\title{
Reduced representation optical methylation mapping $\left(R^{2} O M^{2}\right)$.
}

\author{
Assaf Grunwald ${ }^{1}$, Hila Sharim ${ }^{1}$, Tslil Gabrieli $^{1}$, Yael Michaeli ${ }^{1}$, Dmitry Torchinsky ${ }^{1}$, Rani Arieli ${ }^{1}$, \\ Matyas Juhasz ${ }^{2}$, Kathryn R Wagner ${ }^{3}$, Jonathan Pevsner ${ }^{3}$, Jeff Reifenberger ${ }^{4}$, Alex R Hastie ${ }^{4}$, \\ $\mathrm{Han} \mathrm{CaO}^{4}$, Elmar Weinhold ${ }^{2 *}$, Yuval Ebenstein ${ }^{1^{*}}$. \\ ${ }^{1}$ School of Chemistry, Center for Nanoscience and Nanotechnology, Center for Light-Matter \\ Interaction, Raymond and Beverly Sackler Faculty of Exact Sciences, Tel Aviv University, Tel \\ Aviv, Israel, ${ }^{2}$ Institute of Organic Chemistry RWTH Aachen University D-52056 Aachen \\ Germany, ${ }^{3}$ Kennedy Krieger Institute and Departments of Neurology and Neuroscience, The \\ Johns Hopkins School of Medicine, Baltimore, MD, USA ${ }^{4}$ Bionano Genomics, Inc., San Diego, \\ CA, USA.
}

\section{ABSTRACT}

Reduced representation methylation analysis utilizes a subset of $\mathrm{CpGs}$ in order to report the overall methylation status of the probed genomic regions. Here, we use this concept in order to create fluorescent optical methylation profiles along chromosomal DNA molecules for epigenetic profiling. Reduced representation optical methylation mapping $\left(\mathrm{R}^{2} O \mathrm{M}^{2}\right)$ in combination with Bionano Genomics next generation genome mapping (NGM) technology provides a hybrid genetic/epigenetic genome map of individual chromosome segments spanning hundreds of kilobase pairs (kbp). These long reads, along with the single-molecule resolution, allow for epigenetic variation calling and methylation analysis of large structural aberrations such as pathogenic macrosatellite arrays not accessible to single-cell next generation sequencing (NGS). We show that in addition to the inherent long-read benefits of $\mathrm{R}^{2} \mathrm{OM}^{2}$, it provides genomic methylation patterns comparable to whole genome bisulfite sequencing (WGBS) while retaining single-molecule information. The method is applied here to detect methylation along genes, around regulatory histone marks and to study facioscapulohumeral muscular dystrophy (FSHD), simultaneously recording the haplotype, copy number and methylation status of the disease-associated, highly repetitive locus on chromosome 4q.

\section{INTRODUCTION}

DNA methylation, specifically methylation of the 5-carbon of cytosine, is the most studied and among the most significant epigenetic modifications ${ }^{1}$. In mammalian DNA, methylation mostly occurs on cytosines within CPG dinucleotides (DNA motifs where the cytosine is followed by a guanine residue) that are methylated to an extent of $\sim 70 \%{ }^{2}$. Approximately $60 \%$ of human gene promoters contain clusters of $\mathrm{CpGs}$ referred to as $\mathrm{CpG}$ islands $(\mathrm{CGIs})^{3}$. 
CpG methylation plays an important role in regulation of gene expression with the general notion that hypermethyation of promoters suppresses gene expression ${ }^{4}$. Thus, the methylation status of gene promoters may predict gene activity and relate epigenetic transformations in development and disease to gene expression and protein abundance.

Another genetic feature regulated by $C p G$ methylation is repetitive arrays. These variable copy number elements are homologous DNA sequences, exhibiting identity (or great similarity) to each other ${ }^{5,6}$. Many repetitive elements are mobile and can transpose across the genome, perform homologous recombination events, and promote dynamic genomic transformation ${ }^{6-8}$. This unstable nature explains their size variability, both among different individuals and between different cells of the same individual ${ }^{6,9,10}$. Typically arrays are characterized by the number of units composing them, which has been shown to affect their activity $^{11}$. Methylation of repeat units adds another dimension of variability to these elements: Locally it may regulate the function of individual units and globally it can change the effective number of units in an array altering its activity. In this context, it has been shown that methylation levels of repetitive DNA can regulate repeat-related genetic diseases $^{12,13}$, and are correlated with various types of cancer and their severity ${ }^{14}$. One striking example of a repeat array, addressed in this work, is facioscapulohumeral muscular dystrophy (FSHD), one of the most commons forms of muscular dystrophy, affecting approximately 1 in 7,000-20,000 individuals ${ }^{15-18}$.

The "gold-standard" method for studying CpG methylation is bisulfite sequencing, often used for whole-genome, base-pair resolution methylation profiling by next generation sequencing (WGBS). As such, it provides an averaged representation of the sample's DNA sequence, with distinction between methylated and non-methylated cytosines ${ }^{19}$. The main shortcomings of bisulfite sequencing are the high cost and the requirement for large amounts of starting material due to chemical degradation of the DNA, and the need to split the sample for sequencing the treated and untreated samples separately.

Reduced representation bisulfite sequencing (RRBS) utilizes restriction enzymes that cut at CPG sites, thus specifically enriching for fragments that end within CpG islands. The fragment ends are ligated to adapters, size selected, bisulfite converted, and sequenced to give a representative genome-scale methylation profile. Using the restriction enzyme Mspl to cut DNA at CCGG sites captures $60 \%$ of gene promoters, thus producing important regulatory information while requiring very little input sample ${ }^{20}$. The low input implies that fewer reads are required for accurate sequencing, allowing for high throughput, low-cost methylation analysis for clinical and single-cell applications ${ }^{21,22}$. Nevertheless, bisulfite sequencing and RRBS have limited accessibility to repetitive regions due to the inherent limitations of short-read sequencing, and are often unable to quantify the length and arrangements of the repeats ${ }^{23}$ (Figure S2). Thus, in many cases the genetic and epigenetic characteristics of repetitive elements are still unknown. With this in mind, it is possible that some of the many NGS-inferred sequence duplications are in fact longer repetitive elements. Moreover, since both repetitive elements and methylation patterns tend to display somatic mosaicism, manifesting different structures in different cells ${ }^{10}$, their variability is often masked in the averaged NGS results. 
Here we harness the reduced repre ${ }^{10}$ sentation concept in order to access the methylation profile of long individual chromosome segments by optical genome mapping. Reduced representation optical methylation mapping $\left(\mathrm{R}^{2} \mathrm{OM}{ }^{2}\right)$ seeks to simultaneously capture large scale structural and copy number variants together with their associated methylation status.

DNA optical mapping ${ }^{24-28}$ stands out as an attractive approach for studying large genomic rearrangements such as repeat arrays. The approach consists of a set of techniques for stretching long genomic fragments, followed by imaging of these fragments using fluorescence microscopy. Image processing is used to read out a fluorescently labeled barcode along the molecules that provides genetic information such as the genomic locus of the imaged molecule as well as the size and number of large repeat units. The most advanced method for optical genome mapping involves linearizing and uniformly stretching of fluorescently barcoded DNA molecules in highly-parallel nanochannel arrays. This technique, commercialized by Bionano Genomics Inc., is capable of large scale genetic mapping and automated copy number analysis on a genome scale $e^{29,30}$.

The use of fluorescence microscopy presents the potential of obtaining several types of information simultaneously from the same DNA molecule by using different colors. Labeling different genomic features allows studying epigenetic marks and DNA damage lesions in their native genomic context on the single-molecule level ${ }^{31-33}$. In order to facilitate such multiplexing, we have developed an enzymatic labeling reaction that can distinguish methylated from non-methylated cytosines, and showed that it can be used to detect and quantify methylation levels in synthetic DNA molecules translocated through solid-state nanopores ${ }^{34}$. The bacterial methyltransferase M.Taql in combination with a synthetic cofactor analogue can fluorescently label the adenine residue in TCGA sites containing nonmethylated CpGs. M.Taql has potential access to about 5.5\% of all $\mathrm{CpGs}$, however, these sites represent over $90 \%$ of gene promotors and hence M.Taql is a good candidate for reduced representation methylation profiling and for studying genomic regulatory function. Combining this profile with a genetic pattern generated by labeling of specific sequence motifs, results in a hybrid genetic / epigenetic barcode for every DNA molecule. Using the genetic labels for mapping to the genome reference allows, for the first time, simultaneous assignment of the epigenetic labels to their specific genomic locations, and the study of DNA methylation patterns over large genomic fragments at single genome resolution.

We use $\mathrm{R}^{2} \mathrm{OM}^{2}$ to compare the methylation levels of primary white blood cells and a BLymphocyte derived cell line [NA12878], directly quantifying the reduction in CpG methylation levels after Epstein-Barr virus cell-line transformation. Furthermore, we show that optical methylation profiles are well correlated with whole genome bisulfite sequencing (WGBS) results and give good representation of the methylation status around genes and regulatory elements. In particular, optical signal levels from promotor methylation islands correlate with published gene expression data ${ }^{35,36}$. Finally, we demonstrate the utility of $\mathrm{R}^{2} \mathrm{OM}^{2}$ to simultaneously probe copy number and methylation level in macrosatellite arrays, that when contracted can result in disease, serving as a potential diagnostic assay for FSHD. 


\section{RESULTS}

\section{Methylation sensitive optical mapping quantifies genome-wide and locus specific methylation levels}

The DNA methyltransferase M.Taql methylates the adenine at TCGA sites. The enzyme may be "tricked" to incorporate a fluorophore instead of a methyl group by using a synthetic cofactor analogue ${ }^{37-39}$ (Figure S1a upper panel). However, the M.Taql recognition site (TCGA) contains a nested $\mathrm{CpG}$ dinucleotide, which when methylated blocks the M.Taql reaction ${ }^{40}$.

We first tested whether this methylation sensitive labeling reaction may be combined with optical mapping for studying the methylation levels of various genomes and specific DNA regions. We chose to compare primary blood cells to an Epstein-Barr virus (EBV) transformed blood lymphocyte cell-line, NA12878 (Coriell Institute, USA), where reduced genome-wide methylation levels have been reported ${ }^{41}$.

Genomic DNA was first nick-labeled with the nicking enzyme Nt.BspQI, to create a distinct genetic pattern of red labels along the DNA, regardless of the epigenetic state of the sample $^{42}$. Next, a second layer of information was added by labeling only non-methylated CpGs with a green fluorophore using M.Taql (Figure S1). The labeled DNA was loaded into nanochannel array chips, for dual-color optical genome mapping (Figure 1a). The genetic barcode (red labels) was used to align the molecules to their corresponding sequence locations. After alignment, the epigenetic labels were used to generate $\mathrm{R}^{2} O \mathrm{M}^{2}$ profiles, displaying levels of non-methylated $C P G$ sites along the human genome, with resolution of $1 \mathrm{kbp}$ (Figure 1c). We used the IrysView software suite (Bionano Genomics Inc.) for automatic label detection and genomic alignment of the imaged molecules. Our goal was to provide global quantification of the relative methylation levels in the two samples. As expected $^{41}$, EBV immortalized cell line genomes were hypomethylated compared to DNA from primary cells with a 3.6 fold higher number of M.Taql labels compared to the primary DNA (16.0 Vs. 4.7 labels per $100 \mathrm{kbp}$; Figure 1b). These results demonstrate the utility of M.Taql labeling for quantitative assessment of methylation levels in various samples. 
a.

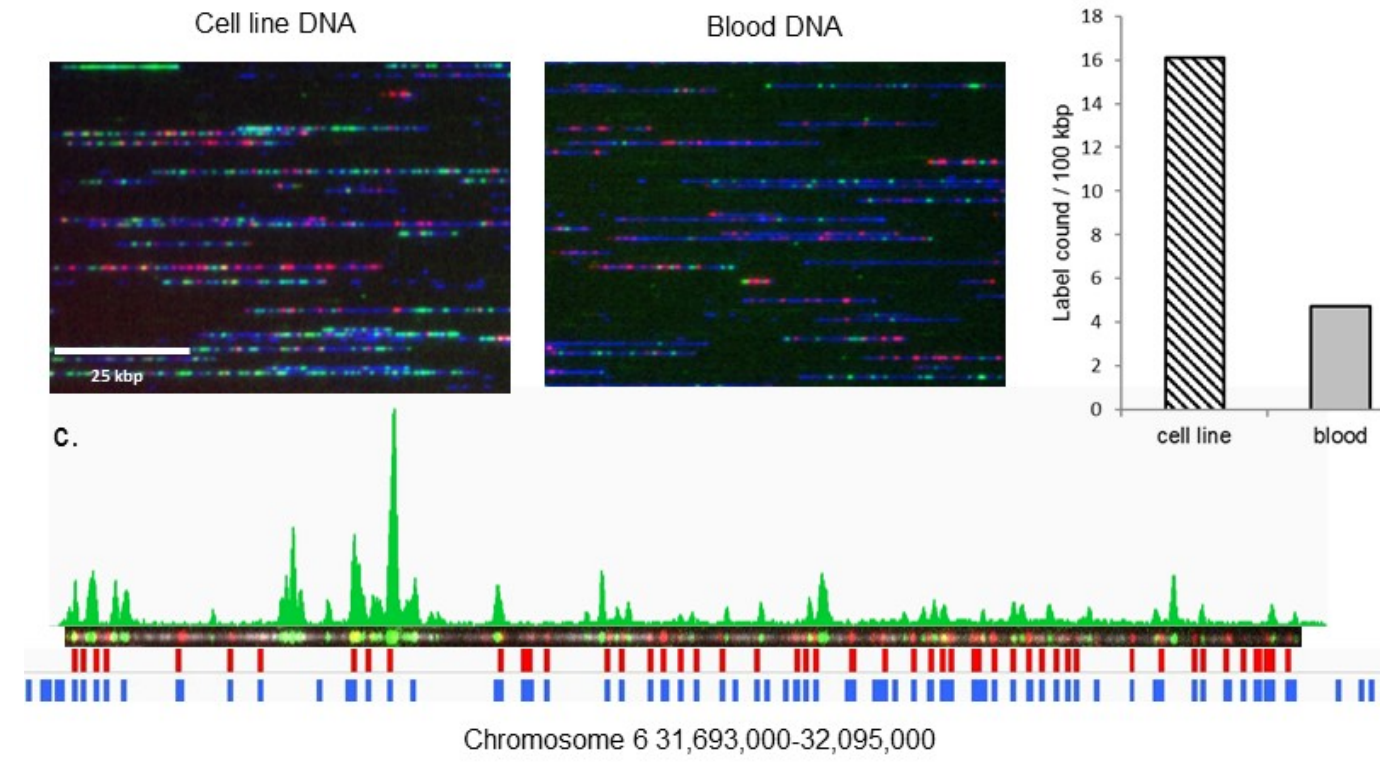

Figure 1. Methylation detection on genomic DNA. a. Genomic DNA from human lymphocyte cell line culture (left image) or primary human blood cells (right image) was dual labeled with genetic labels (Nt.BspQl, red labels) and methylation sensitive labels (M.Taql, green labels), and imaged on a nanochannel array chip. Representative images from both samples are presented here. DNA backbone, stained with YOYO-1 is displayed in blue. b. Nonmethylated CpG labels were detected for both DNA samples. The bar-graph presented here displays number of detected labels per $100 \mathrm{kbp}$ (in total $4.6 \mathrm{Gbp}$ were sampled for the primary blood cells DNA and $1.2 \mathrm{Gbp}$ for the lymphocyte cell line). Dashed bar displays label number for the cell line DNA and gray bar for the primary blood DNA . It is clear that epigenetic labels are more frequent in the immortalized cell-line genome, indicating that it is relatively non-meth ylated as expected. c. A representative dual labeled molecule from the primary blood sample. $\mathrm{R}^{2} \mathrm{OM}^{2}$ profile along the molecule is presented in green above the molecule image. The detected locations of genetic labels are shown in red below the image, and the reference barcode, used for alignment to the locus in chromosome 6 is shown in blue.

\section{$R^{2} O M^{2}$ provides genome-wide methylome profiles of genes and regulatory elements}

We validated the quality of $\mathrm{R}^{2} O \mathrm{M}^{2}$ profiles by correlating our results to available "gold standard" WGBS data. Levels of non-methylated CpGs were calculated along gene bodies and promotor regions and around four commonly used regulatory histone marks (Figure 2). In all cases the results showed high correlation with WGBS data and is in agreement with the expected methylation status of these regions ${ }^{43}$. Additionally, we found that on a genome scale, $\mathrm{R}^{2} O \mathrm{M}^{2}$ profiles correlate well with WGBS profiles, especially given the inherent $5.5 \%$ sampling rate (Figure $2 \mathrm{C}$ ). 


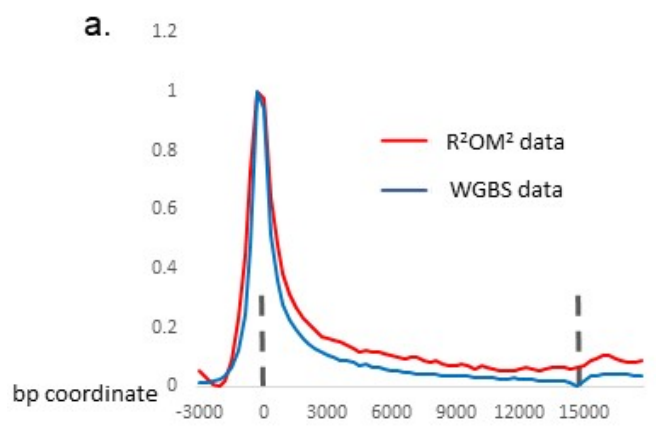

a. 1.2

c.

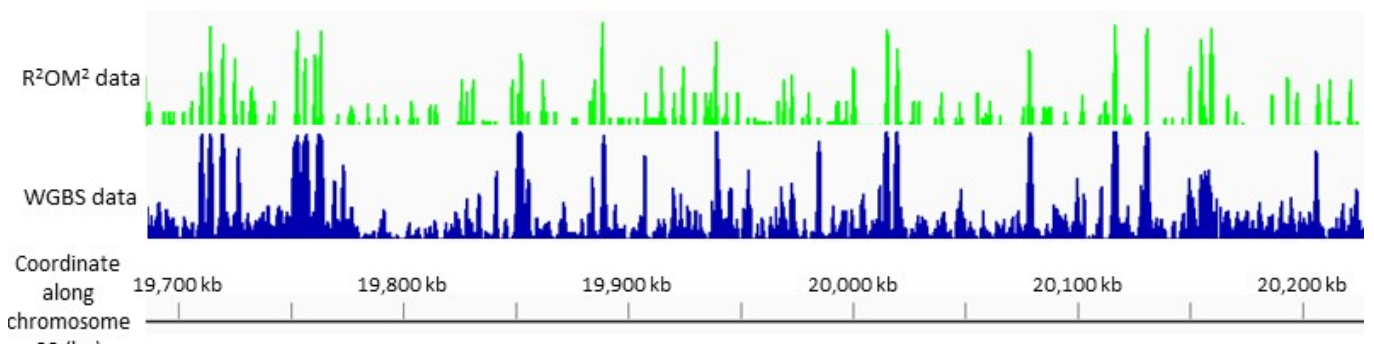

22 (bp)

Figure 2. $\mathrm{R}^{2} \mathrm{OM}$ profiles correlate with WGBS based profiles. a. Non-methylated $\mathrm{CpG}$ levels along gene bodies for $\mathrm{R}^{2} \mathrm{OM}^{2}$ data (red) and WGBS data (blue). For both data types, non-methylated CpG levels were sampled along each gene separately and the plot displays average and normalized nonmethylated $\mathrm{CPG}$ values for all genes in the human genome. The presented region spans gene body and $3 \mathrm{kbp}$ up and downstream from the transcription start and end sites (indicated by dashed lines at the left and right rims of the track respectively). It is clear that both methods produce highly similar tracks reporting depleted methylation around the transcription start sites as expected. $\mathbf{b}$. Plots of meth ylation levels spanning $6 \mathrm{kbp}$ around four regulatory histone marks. For each histone modification, the genome-wide average methylation values are plotted for each modification type: H3K4me3 (red), H3K9me3 (green), H3K4me3 (purple), H3K9ac (blue). For the sake of demonstration, the WGBS plots are inverted so that they appear below the $\mathrm{R}^{2} \mathrm{OM}^{2}$ data. It is clear that both data types produce similar results not only in the shape of non-methylation profiles around the regulatory sites but also in their intensities. c. Non-methylated CpG levels as detected along the human genome: $\mathrm{R}^{2} \mathrm{OM}^{2}$ (green track) and WGBS (blue track). A region of about $500 \mathrm{kbp}$ is presented to allow sufficient resolution (the presented region was randomly chosen along chromosome 22 and reflects the overall correlation).

The main advantage of $R^{2} O M^{2}$ is the ability to generate extremely long reads from individual molecules, thus, allowing for direct comparison between long methylation patterns ranging hundreds of kbps. This is exemplified in Figure 3 showing $\mathrm{R}^{2} \mathrm{OM}^{2}$ profiles from genomes of three primary white blood cells aligned to the same $250 \mathrm{Kbp}$ genomic region in chromosome $1 \mathrm{p}$. The $\mathrm{R}^{2} \mathrm{OM} \mathrm{M}^{2}$ profiles are presented below the calculated distribution of $\mathrm{CpG}$ and M.Taql sites in this region and the positions of associated genes are presented in the bottom panel. The molecules in Figure $3 d$ exhibit similar methylation profiles that are well correlated with positions of calculated CpG islands. Small variations in the pattern are seen and can be attributed to common variation in methylation status among white blood cells ${ }^{44}$. Furthermore, the maps allow relating promoter methylation to gene expression data. For 
instance, a CpG island that lies in the promotor region of the PHF13 gene (indicated by a red box), was found to be non-methylated by both sequencing and $\mathrm{R}^{2} O \mathrm{M}^{2}$, and is known to be expressed in white blood cells ${ }^{36}$. In contrast, the promoter of the $K L H L 41$ gene (indicated by a black box), was detected as methylated by both methods. This muscle specific gene is indeed expected to be silenced in blood cells ${ }^{35}$. These findings and their agreement with established NGS results validate the ability of $R^{2} O M^{2}$ to unravel the methylome at a singlecell-like level. Optical mapping allows genomic information to be read directly from individual, un-amplified, long fragments of DNA, thus mitigating the bias of large structural and copy number variation in single cell sequencing experiments.

a.

Chromosome 1
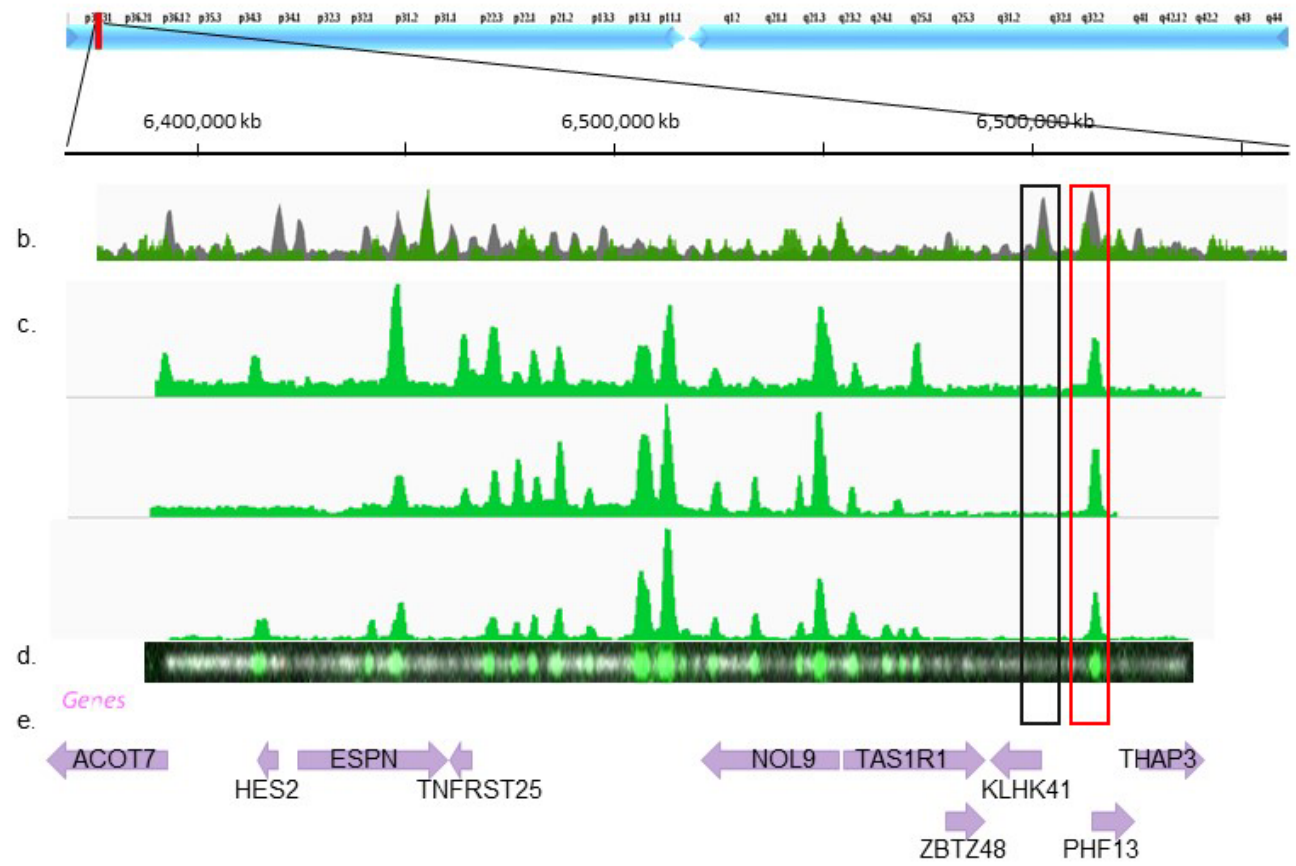

Figure 3. a. Detailed view of chromosome 1:6,350,000-6,670,000 bp. b. A histogram of CpG (gray) and M.Taql (green) sites across the region. c. Digital representation of three detected molecules aligned to the reference sequence based on Nt.BspQl labels $\left(\mathrm{P}\right.$-value $<10^{-20}$ ). Label intensity is shown in green. e. The raw image of the backbone (light gray) and the M.Taql channel (green) of the bottom molecule presented in d. f. Gene body locations and corresponding HGNC gene symbols. Each gene is represented as a purple arrow and the direction of the arrow indicates gene orientation. Black and red rectangles across b-e indicate methylated and nonmethylated gene promoters, respectively.

\section{Simultaneous quantification of copy number and methylation state in DNA tandem repeats}

Macrosatellite arrays, repetitive DNA that spans up to millions of base pairs across the genome, are extremely challenging for analysis by next-generation sequencing. Analysis is 
further complicated by the recent understanding that DNA methylation plays a crucial role in the function of these regions. One striking example of the significance of methylation in such regions is the D4Z4 array on chromosome 4, which is directly related to the muscular dystrophy FSHD ${ }^{45}$. Recent evidence show that both the number of D4Z4 repeats and their methylation status constitute the genotype of the disease, dictating whether the individual manifests disease symptoms or not. Commonly, healthy individuals carry an array of more than 10 repeats. However, even long arrays result in FSHD symptoms when losing their methylation (termed FSHD2) while carriers of short but highly methylated repeat arrays, do not manifest the disease ${ }^{15,16}$. The multiple combinations of copy number and methylation level span a broad range of possible variations which are correlated with the disease severity and manifestation ${ }^{16,17}$.

To demonstrate the capabilities of optical mapping for simultaneous copy number quantification and DNA methylation detection, we studied a model system of FSHDassociated D4Z4 repeats from healthy human individual cloned into the $\mathrm{CH} 16-291 \mathrm{~A} 23$ bacterial artificial chromosome (BAC) (Figure 4a). We first attempted to evaluate the copy number of the studied array by NGS read-depth analysis ${ }^{46}$. We reasoned that the ratio between number of reads representing the repeat unit, relative to the number of reads detected for a single copy region, would provide the array copy number. Purified BAC DNA was sequenced using Illumina MiSeq to a read depth of 15,000X. We used read depth analysis to assess the D4Z4 copy number. Briefly, all sequencing reads were aligned to the BAC's reference sequence, containing only one repeat, and the copy number was calculated as the ratio between the number of reads ("coverage") aligned to the repetitive and the non-repetitive regions respectively. The coverage along the non-repetitive sequence displayed variation of up to $25 \%$, while the coverage along the repeat sequence was extremely variable, averaging $63 \%$ of the mean read depth (Figure S2). The ratio between the median coverage value along the repetitive and non-repetitive sequences was $\sim 8$, implying that this is the number of D4Z4 repeats along the BAC (Figure 3c). However, the large standard deviation values strongly demonstrate the unreliability of this method, as well as the sensitivity of PCR amplification and NGS to the exact content of the investigated sequence. 


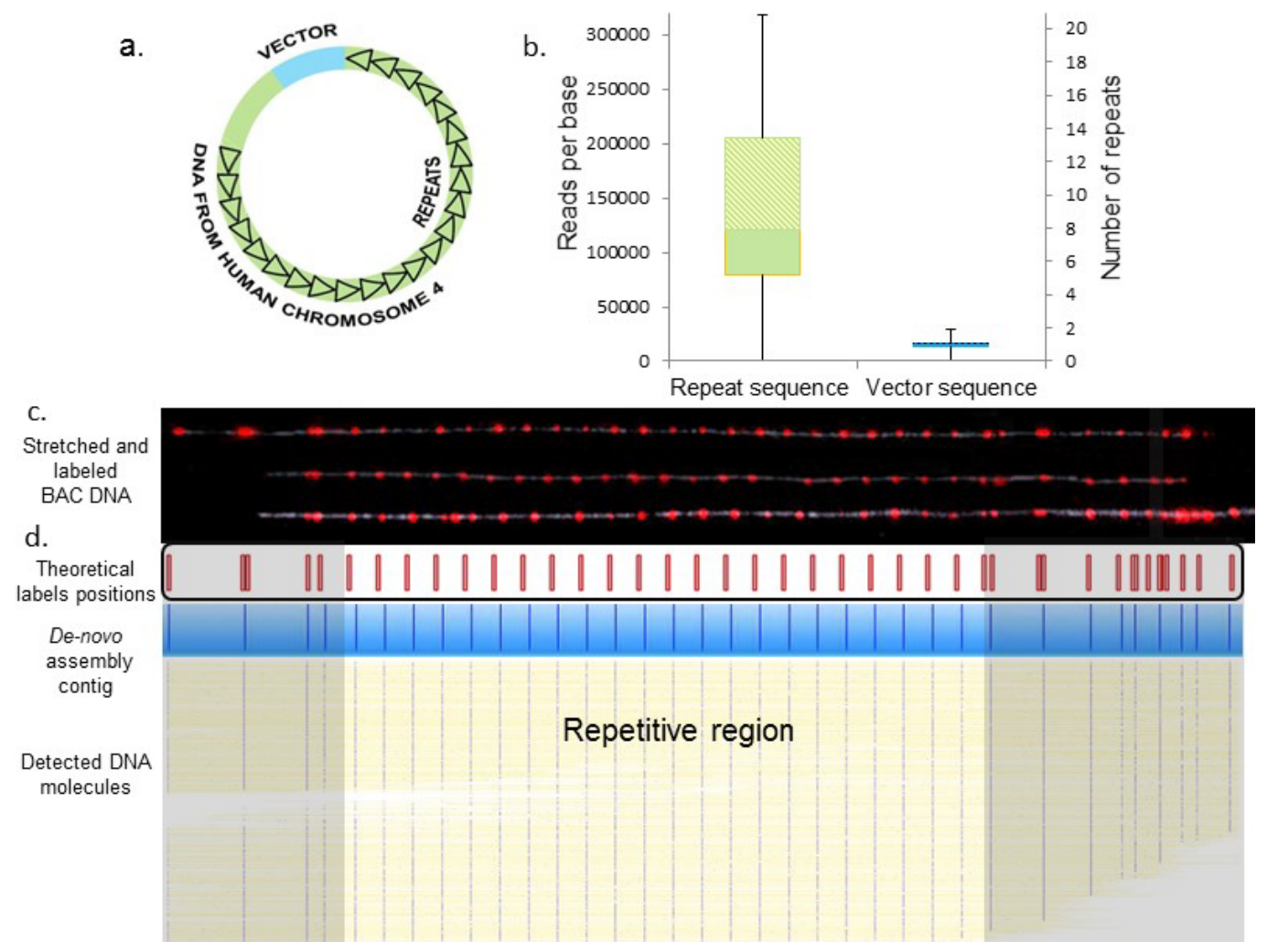

Figure 4. Copy number analysis. a. The FSHD BAC model system contained an unknown number of D4Z4 repeats (black triangles), an unknown size of genomic DNA 5 ' to the repeat array (green), and the cloning vector (blue). $\mathbf{b}$. Read depth analysis box plots displaying the 25 th percentile (bottom of the lower box), the median (intermediate between boxes) and the 75th percentile (top of the upper box), for coverage values of the sequencing reads along the repeat region (left box), and the non-repetitive region (including the vector and the upstream to repeat region, right box). The scale on the right is normalized to the median coverage along the non-repetitive region. $c$. Representative images of three intact model molecules labeled with $\mathrm{Nb}$.Bsml (red dots) and stretched on modified glass surfaces. The pattern of labeled molecules can be aligned to the reference map presented below the images (expected labeling locations are shown in red). The repetitive region can be distinguished by virtue of the equally spaced labels, each representing a repeat, and quantified by simply counting the labels ( 23 repeats). d. 627 digital representations of labeled DNA molecules stretched and imaged in a nanochannel array chip. The detected molecules were de-novo assembled into a single consensus map (displayed in blue) which shows excellent agreement with the theoretical map. The yellow lines represent the DNA backbone and the blue dots represent the detected labels. The assembled map contained 23 repeat units.

Next, we directly visualized the repeats along stretched DNA molecules. We used tailored labeling of sequence motifs contained in the repetitive element to highlight individual repeats and allow for physical counting of the copy number. We nick-labeled the BAC DNA using nicking enzymes ( $\mathrm{Nb}$. Bsml or $\mathrm{Nb}$.BssSl) which have a single recognition site on the 3.3 $\mathrm{kbp}$ long D4Z4 repeat sequence, yielding a single distinct fluorescent tag for each repeat unit. The labeled DNA was stretched and immobilized for visualization on modified glass slides, using a simple micro-fluidic scheme (see supporting information). This method allowed fluorescence imaging of the entire DNA contour and localization of individual fluorescent labels along the DNA (Figure 4c). The same sample was loaded onto an Irys instrument (Bionano Genomics Inc., CA. USA), which facilitates high-throughput DNA stretching and imaging in nanochannel array chips. The post-imaging analysis, performed by the IrysView software suite, involved automatic label detection and de-novo assembly of the molecules into a contiguous consensus barcode. The resulting consensus map was created in 
an unsupervised manner based on label patterns from approximately one thousand detected molecules ${ }^{47,48}$. When comparing the non-repetitive region of this contig to the one predicted from the known sequence, an almost perfect match was obtained ( $p$-value $<10^{-43}$, Figure $4 \mathrm{~d}$ ). The consensus repetitive region is unambiguously composed of 23 D4Z4 repeats, demonstrating the potential of the technique for genetic diagnosis of FSHD.

For methylation analysis, we performed $\mathrm{R}^{2} \mathrm{OM}^{2}$ as an overlay on the repetitive genetic barcode. We used red fluorophores for the genetic barcode and green fluorophores for methylation mapping. Figure 5a shows the unique pattern created by M.Taql along the nonmethylated $\mathrm{BAC}^{49}$, highlighting non-methylated repeat units. M.Taql has two close-by recognition sites on each repeat unit that result in one visual label on each repeat, due to diffraction limits. Nevertheless, when over-stretching the non-methylated BAC on modified microscope slides, the two green methylation labels flanking the red genetic label were clearly resolved, in agreement with the theoretical dual-color barcode (Figure 5b).

To simulate the native state of DNA, where repeat arrays are methylated to variable degrees, we partially methylated the DNA using the CpG-specific DNA MTase M.ssSI. We repeated the dual-labeling reaction on the partially methylated sample, as well as on a nonmethylated control, and analyzed both on the nanochannel array chips. After image analysis, we used the red genetic labels for automated de-novo assembly and generated the consensus map with the 23 repeats as described earlier. With thousands of molecules now aligned to the consensus map, we could compare the green methylation patterns generated by $\mathrm{R}^{2} \mathrm{OM}^{2}$ on the non-methylated and partially methylated samples. Figure $5 \mathrm{c}$ shows integrated methylation profiles generated from the molecules detected in each data set. The non-methylated sample shows frequent labeling, at the expected locations (green dots), while the partially methylated DNA sample shows significantly fewer labels (blue dots). It is clear from the integrated plot that in the partly methylated sample, methylated CpGs are distributed equally among the repeat units, in line with the fact that the partial methylation was random and equal for all repeats in the array. These results clearly demonstrate that $\mathrm{R}^{2} \mathrm{OM}^{2}$ enables not only single-molecule and single-repeat resolution but also assessment of the average methylation status for each individual repeat in the array across a population of different DNA molecules. 
bioRxiv preprint doi: https://doi.org/10.1101/113522; this version posted November 16,2017 . The copyright holder for this preprint (which was not certified by peer review) is the author/funder, who has granted bioRxiv a license to display the preprint in perpetuity. It is made available under aCC-BY-NC 4.0 International license.

a.

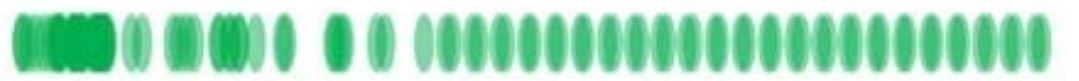

b.
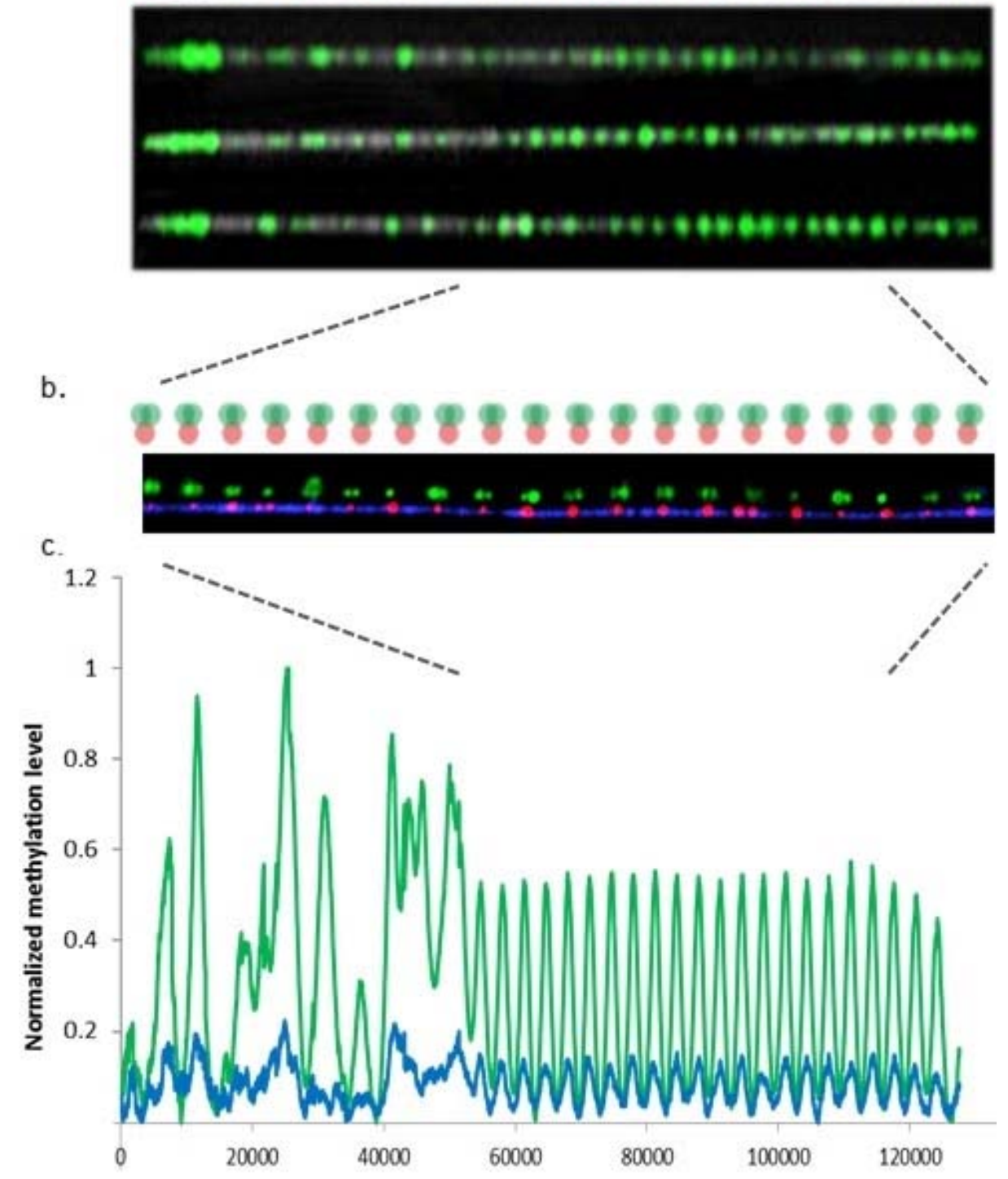

Figure 5. a. A reference map simulating the relative expected locations of $R^{2} O M^{2}$ labels generated by $M$.Taql along the stretched BAC (green). Below, images of three non-methylated, M.Taql labeled BAC molecules linearized and stretched in nanochannels and aligned to the reference map. b. Partial genetic map (red) and $\mathrm{R}^{2} \mathrm{OM}^{2}$ (green) from the repetitive region of the FSHD BAC stretched on a modified glass slide. The genetic identity and the number of repeat units are highlighted by labeling with the nicking enzyme Nb.Bsml (red labels). Co-labeling the DNA with M.Taql (green labels) indicated that the molecules were non-methylated. The displayed image is an overlay of red and green channels along the repetitive region of a single BAC molecule. Above the repeats is the reference map for the region. The green channel is shifted upwards to allow better visualization. The higher stretching factor achieved on modified glass compared to nanochannels, enables detection of the two M.Taql labels flanking the genetic label in each repeat. c. Comparative $\mathrm{R}^{2} \mathrm{OM}^{2}$ of non-methylated and partiallymethylated BAC samples. Normalized integrated maps of detected M.Taql labels are presented for the nonmethylated (green, 18074 molecules) and partially-methylated (blue, 9089 molecules). Both plots highly correlate with the expected reference map. 


\section{$R^{2} O M^{2}$ enables genetic / epigenetic diagnosis of FSHD}

Finally, we performed $\mathrm{R}^{2} \mathrm{OM}^{2}$ on whole blood DNA extracted from a donor previously diagnosed with FSHD via commercial testing based on pulsed-field gel electrophoresis and Southern blot. Figure 6 shows the genome reference barcode (green bar) and the two haplotypes detected in the sample (blue bars). Genetic labeling allowed distinguishing between the two very similar $4 \mathrm{qA}$ and $4 \mathrm{qB}$ alleles, each with a distinct copy number of D4Z4 repeats. Macrosatellite repeats were visually marked by the equally spaced methylation sensitive labels (marked green) indicating a non-methylated array. We measured 4 repeat blocks for the $4 q A$ allele and 8 repeat blocks on the non-pathogenic $4 q B$ allele $e^{50}$. This was similar to previous genetic assessments based on clinically performed pulsed-field gel electrophoresis in which the EcoRI/BInl allele of $15 \mathrm{~kb}$ on chromosome $4 \mathrm{qA}$ corresponding to approximately 4 repeats and the $4 q B$ allele was estimated to have a EcoR1/BInl allele size of $27 \mathrm{~kb}$, corresponding to approximately $7 \mathrm{D} 4 \mathrm{Z4}$ repeats. These data demonstrate the utility of this optical mapping approach for characterizing the genetic and epigenetic profile of FSHD as well as other macrosatellite arrays and large structural variants.

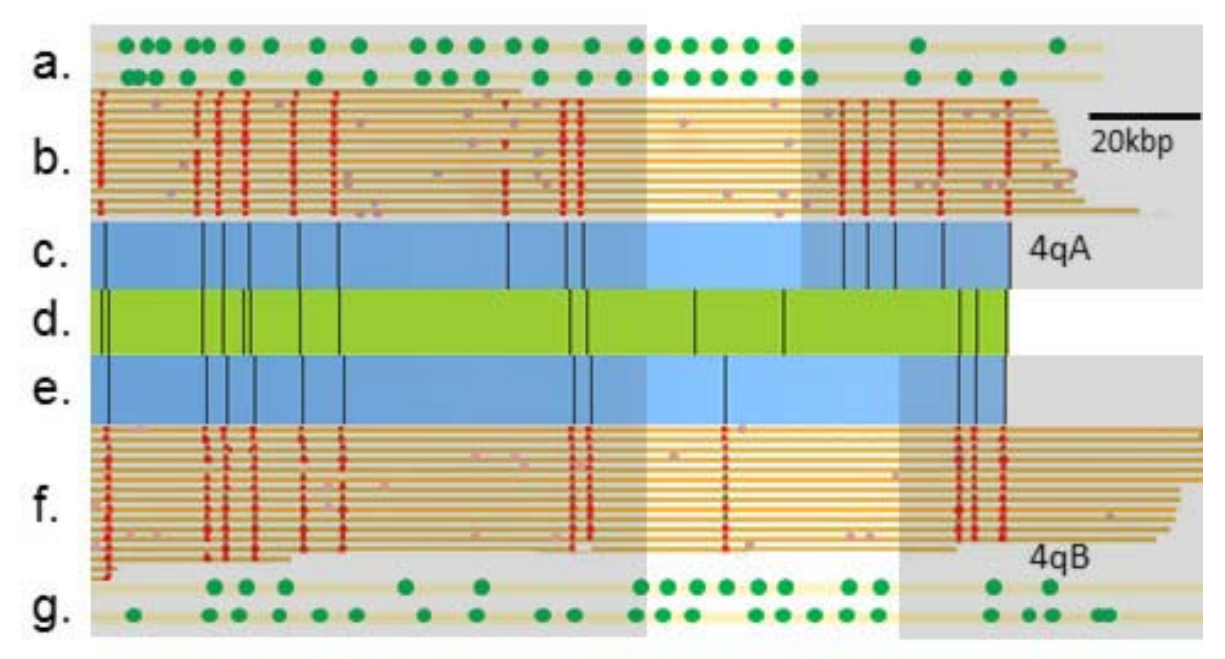

Figure 6. Structural variation analysis and methylation analysis of the pathogenic contraction on chromosome $4 q$ of a patient with FSHD. The central green horizontal bar (d.), shows the reference map for chromosome 4q. The consensus optical maps of the two haplotypes, $4 \mathrm{qA}$ (c.) and $4 \mathrm{qB}$ (e.), generated by De-Novo assembly are above and below the reference. $\mathbf{b}$./f. show genetic maps generated from individual molecules aligned to the genome reference. Maps were clustered by haplotype (4qA and $4 q B)$. Red dots represent labels used for alignment. a./g. show $\mathrm{R}^{2} \mathrm{OM}{ }^{2}$ profiles of DNA molecules aligned to the $4 \mathrm{qA} / 4 \mathrm{qB}$ allele and displaying hypomethylation of the D4Z4 repeats. The gray background covers the non-repetitive region on each allele.

\section{DISCUSSION AND OUTLOOK}


Optical genome mapping has recently been used to map whole human genomes at high coverage and to highlight genetic variability between individuals with unprecedented detail $^{29,30}$. This work adds an epigenetic component to optical maps, providing, for the first time, correlated genetic/epigenetic profiles for individual DNA molecules spanning hundreds of thousands of base pairs. Utilizing state-of-the-art optical genome mapping technology, combined with DNA MTase-assisted methylation detection, we create a reduced representation optical methylation map, $\mathrm{R}^{2} O \mathrm{M}^{2}$, which reports on the methylation status of $90 \%$ of gene promoters. The reduced representation provides sufficiently sparse labeling to comply with the low resolution of optical maps while providing high correlation with BS-seq data around regulatory elements. We demonstrate automatic, high-throughput analysis of global methylation levels in various cell types by quantifying the amount of non-methylated $\mathrm{CpGs}$. Additionally, $\mathrm{R}^{2} \mathrm{OM}^{2}$ provides long read / low-resolution methylation profiles at different genomic loci with emphasis on CPG islands and regulatory sequence elements such as gene promoters and histone binding sites. This property may allow studying the epigenetic profiles of genes together with remote regulators such as distant enhancers and cis elements. One area in which $\mathrm{R}^{2} \mathrm{OM}^{2}$ has a particular advantage over existing technologies is the study of DNA methylation of large structural variants and repetitive arrays. DNA repeats are dynamic regions, exhibiting high variability both in length and methylation status, and thus may differ significantly between individuals ${ }^{9,51}$. Today, in the era of personalized medicine, it is becoming increasingly accepted that the full profile of genomic structural variation, including DNA repeats, is directly linked to health and susceptibility to disease ${ }^{8,52} \cdot \mathrm{R}^{2} \mathrm{OM}^{2}$ offers single-molecule level information on the size of the region, the number of repeat units, and the methylation status of individual repeats. This detailed information is inaccessible via current genome technologies such as NGS, DNA arrays, and quantitative PCR, which mostly provide averaged or inferred data and cannot specifically address individual repeat units.

We demonstrate the utility of $\mathrm{R}^{2} \mathrm{OM}^{2}$ for characterizing the D4Z4 repeat array, where both the size and the methylation status of the repeats affect FSHD disease manifestation ${ }^{16}$. Specifically, the reported approach can distinguish between healthy individuals, FSHD1, and FSHD2 carriers by combining copy number and methylation level information. Moreover, the detailed view of the methylation status of individual repeats may offer new insights into the mechanism of disease and may lead to a more individualized prognosis than is offered by current commercial testing. Notably, the $\mathrm{R}^{2} \mathrm{OM}^{2}$ concept is not limited to D4Z4 repeats, and various labeling enzymes may be used to address different target sequences, thereby extending the repertoire of available targets for this method. Further development of this technique may serve to map differentially methylated single-molecule patterns on a genome-wide scale, potentially allowing simultaneous genetic/epigenetic haplotyping, as well as ultra-sensitive detection of epigenetic transformations.

\section{ONLINE METHODS}

\section{Human Subjects}


This study was approved by The Johns Hopkins School of Medicine Institutional Review Board. The donor with a clinical diagnosis of FSHD1 was confirmed by the University of lowa Diagnostic Laboratories to have a contracted D4Z4 array on a 4qA allele by pulse-field gel electrophoresis and Southern blotting. The EcoRI/BInl allele of $15 \mathrm{~kb}$ on chromosome $4 \mathrm{qA}$ corresponds to approximately 4 repeats, and of $27 \mathrm{~kb}$ allele on chromosome $4 \mathrm{qB}$ corresponds to approximately 7 repeats.

\section{DNA samples}

$\lambda$-Bacteriophage DNA (New England BioLabs, Ipswich MA, USA) was used as provided. BAC DNA was purified from E.coli cells containing the $\mathrm{CH} 16-291 \mathrm{~A} 23$ BAC. Cells were cultured overnight in LB containing $12.5 \mu \mathrm{g} / \mathrm{ml}$ cloramphenicol (Sigma-Aldrich, Rehovot, Israel) at $30^{\circ} \mathrm{C}$. BAC DNA was purified from the cells using the NucleoBondXtra BAC kit (MACHEREYNAGEL Inc. Düren, Germany). DNA from a lymphocyte cell line (NA12878, Coriell Institute for Medical Research, Camden New Jersey, USA) ,from primary blood cells (purchased from HemaCare Inc. Van Nuys, CA, USA) and whole blood DNA from human subjects were purified in agarose plugs in order to maintain large DNA fragments, following the Bionano Genomics IrysPrep protocol ${ }^{29}$.

\section{M.Taql - assisted labeling for $\mathrm{R}^{2} O \mathrm{M}^{2}$}

To generate methylation sensitive labels we used the DNA MTase M.Taql, which catalyzes the transfer of a carboxytetramethylrhodamine (TAMRA) fluorophore from the synthetic cofactor AdoYnTAMRA onto the adenine residue within its recognition sequence (TCGA) ${ }^{53}$. The labeling reaction was carried out as follows: $1 \mu \mathrm{g}$ of DNA was reacted with $37.5 \mathrm{ng}$ of M.Taql and $40 \mu \mathrm{M}$ of AdoYnTAMRA in labeling buffer $\left(20 \mathrm{mM}\right.$ Tris-HOAc, $10 \mathrm{mM} \mathrm{Mg}(\mathrm{Cl})_{2}, 50$ $\mathrm{mM} \mathrm{KOAc}, 1 \mathrm{mM}$ DTT, pH 7.9) in the presence of $0.01 \%$ Triton-X 100 and $0.1 \mathrm{mg} / \mathrm{ml} \mathrm{BSA}$ in a total reaction volume of $25 \mu \mathrm{L}$ at $60^{\circ} \mathrm{C}$ for 1 hour. The labeled DNA was reacted with $40 \mu \mathrm{g}$ of proteinase $\mathrm{K}$ (Sigma-Aldrich, Rehovot, Israel) at $45^{\circ} \mathrm{C}$ for 1 hour to disassemble protein-DNA aggregates. For methylated samples $\mathrm{CpG}$ methylation was performed prior to labeling using the CpG-specific DNA MTase M.Sssl (Thermo Scientific, Waltham, MA, USA) according to manufacturer's instructions but with twice the suggested amount of enzyme to ensure complete methylation. To obtain partial methylation, the reaction was carried out using the recommended amount of enzyme, but for $75 \%$ of the recommended incubation time. Methylation was verified by digestion with a methylation-sensitive restriction enzyme Hpall (New England BioLabs Inc., Ipswich MA, USA), followed by gel electrophoresis to ensure that the DNA was protected or partially protected from restriction (Figure S1).

\section{Nick labeling for optical genome mapping}

DNA was prepared in a nick-labeling-repair reaction (NLR) involves (a) the nicking enzyme $\mathrm{Nb.Bsml}$ or Nt.BspQl which generates single-strand nicks at its specific recognition sites (GAATGC or GCTCTTCN respectively), (b) a DNA polymerase enzyme incorporates fluorescent nucleotides at the nicked sites; and finally, (c) a DNA ligase enzyme repairs the 
remaining single-strand breaks. For the NLR reaction involving Nt.BspQI, DNA was labeled using the IrysPrep kit (Bionano Genomics Inc., San Diego CA, USA) according to manufacturer's instructions. For Nb.Bsml-based NLR, DNA (900 ng) was first reacted with 30 units of the enzyme (New England BioLabs Inc., Ipswich MA, USA) in $30 \mu$ l NEBuffer 3.1 for 120 minutes at $65^{\circ} \mathrm{C}$. Next, the DNA was reacted with 15 units of Taq DNA polymerase (New England BioLabs Inc., Ipswich MA, USA) in the presence of the following nucleotides: dGTP, dCTP dATP (Sigma-Aldrich, Rehovot, Israel) and the fluorescent nucleotide dUTP-Atto647 (Jena Bioscience $\mathrm{GmbH}$, Jena, Germany) at a final concentration of $600 \mathrm{nM}$ (each). The reaction was carried out in a reaction buffer (ThermoPol buffer, New England BioLabs Inc., Ipswich MA, USA) in a total volume of $45 \mu$ for $60 \mathrm{~min}$ at $72^{\circ} \mathrm{C}$. Finally, the DNA was reacted with 120 units of Taq DNA ligase (New England BioLabs Inc., Ipswich MA, USA) with $0.5 \mathrm{mM}$ $\mathrm{NAD}^{+}$(New England BioLabs Inc., Ipswich MA, USA), in a reaction buffer (ThermoPol Buffer, New England BioLabs Inc., Ipswich MA, USA) including 0.5 mM NAD+ (New England BioLabs Inc., Ipswich MA, USA) and of $10 \mu \mathrm{M}$ dNTP mix, in a total reaction volume of $60 \mu \mathrm{L}$ for $30 \mathrm{~min}$ at $45^{\circ} \mathrm{C}$. For $\mathrm{R}^{2} \mathrm{OM} \mathrm{M}^{2}$ experiments, DNA was initially labeled by NLR and then $0.05 \mu \mathrm{g}-0.5 \mu \mathrm{g}$ of the labeled DNA was reacted with M.Taql as described above (the reaction was scaled down accordingly). Prior to M.Taql labeling the Nb.Bsml NLR reaction products were reembeded in $1 \%$ agarose plug to allow it's washing in water (plugs were incubated for ten minutes in water, this was repeated three times by replacing the water). In the case of Nt.BspQI-NLR, the M.taql reaction was performed in the NLR buffer with addition of $1 x$ Buffer 4 and 1x BSA (New England BioLabs Inc., Ipswich MA, USA) and Ph was adjusted to 8.3 using $0.1 \mathrm{M} \mathrm{NaOH}$. Before imaging the agarose matrix was digested using GELase enzyme (Epicenter, Madison, WI, USA).

\section{Sample preparation, DNA stretching and imaging}

Post labeling, BAC and $\lambda$ DNA were cleaned by ethanol precipitation as has been described previously ${ }^{53}$. Genomic DNA was cleaned by embedding it into agarose plugs and washing these in TE buffer (see supporting information). Prior to imaging, the labeled DNA was stained with $0.5 \mu \mathrm{M}$ of YOYO-1 (Invitrogen, Carlsbad, CA, USA) for visualization of its contour. DTT (Sigma-Aldrich, Rehovot, Israel) was added to the reaction (200 mM) to prevent photo bleaching and DNA breaks. To stretch the DNA from its random coil conformation into a linear form, allowing imaging of its contour, we used two types of experimental scheme: The first type of stretch used modified glass surfaces. In this approach, DNA sample-solutions were flowed by applying capillary forces or using microfluidics, on glass surfaces that were chemically modified to facilitate the DNA's anchoring and stretching on the surfaces (see supporting information). After stretching, the DNA was imaged using an epifluorescence microscope (FEI Munich GmbH, Germany) equipped with a high-resolution EMCCD IXon888 camera (Andor Technology Ltd. Belfast, UK). A 150 W Xenon lamp (FEI Munich GmbH, Germany) was used for excitation with filter sets of 485/20ex and 525/30em, 560/25ex and 607/36em, and 650/13ex and 684/24em (Semrock Inc., Rochester NY, USA) for the YOYO-1, TAMRA, and Atto-647 channels respectively. For high-throughput mapping experiments, samples were analyzed in nanochannels array chips (Bionano Genomics Inc., San Diego CA, USA). On the chip, DNA is forced into $45 \mathrm{~nm}$ square nanochannels using an electric field and is stretched along the 
channel axis for imaging. This process is carried out in automated cycles by the Irys instrument (Bionano Genomics Inc., San Diego CA, USA) ${ }^{42}$.

\section{Data analysis}

For analysis of the high-throughput nanochannel array data, raw images were processed and DNA molecules were detected and digitized by custom image-processing and analysis software (Irys Extract ${ }^{54}$ or IrysView ${ }^{29}$ ). Genetic labels were assigned one set of coordinates along the molecules (the genetic map) and the methylation labels are assigned another set (the methylation map). One output of the detection process is the number of labels per 100 kbp allowing for direct comparison between labeling levels of different samples, and quantitative assessment of methylation levels (Figure S3).

For $\mathrm{R}^{2} \mathrm{OM}{ }^{2}$, DNA molecules are first mapped to the genome reference based on the match between the fluorescent genetic pattern along the molecule and the pattern expected from the known reference sequence. after alignment, the methylation maps indicated the distribution of non-methylated $\mathrm{CpGs}$ along the mapped genomic regions, and methylation profiles were exported in BED format for visualization in standard genome browsers such as the UCSC genome browser ${ }^{55}$ (https://genome.ucsc.edu/index.html).

Details on bioinformatics analysis and comparison to BS sequencing data is provided in the supporting information

Irys View also enabled de-novo assembly of the detected molecules into a consensus barcode or contig (genome map) and its comparison with the theoretical barcode. This unsupervised assembly was used to assess the number of repeats in the FSHD BAC model system.

Images of DNA molecules stretched on modified glass surfaces were manually aligned, according to the barcode created outside the repetitive region. This enabled detection of the starting point of the repeat array, allowing counting of individual repeat units.

\section{Next-generation sequencing}

Purified BAC DNA was sheared using Covaris AFA (Covaris Inc. Woburn MA, USA) and the fragments were size-separated by electrophoresis using agarose gel, allowing selective extraction of fragments within the range of $150-300 \mathrm{bp}$. Illumina sequencing libraries were prepared using NEXTFlex kit (Bioo Scientific Corporation, Austin TX, USA) and sequenced using MiSeq (Illumina Inc. San Diego, CA. USA) to a paired-end coverage of 15,000X. Sequencing reads were de-novo assembled using CLC Workbench software (CLC Bio-Qiagen, Aarhus, Denmark).

\section{ACKNOWLEDGEMENTS}

We thank Prof. D. Gabellini for his kind gift of the CH16-291A23 BAC and K. Glensk for preparing M.Taql. We acknowledge financial support from the German-Israeli foundation [I- 
1196-195.9/2012] (E.W. and Y.E.), the BeyondSeq consortium [EC program 63489] (Y.E.), the

European Research Councils starter grant [337830] (Y.E.) i-Core program of the Israel Science Foundation [1902/12] ( Y.E.), NIH U54HD060848 (KRW), and NIH U01 MH106884 (JP).

\section{REFERENCES}

(1) Bird, A. DNA Methylation Patterns and Epigenetic Memory. Genes Dev. 2002, $16,6-21$.

(2) Jabbari, K.; Bernardi, G. Cytosine Methylation and CpG, TpG (CpA) and TpA Frequencies. Gene 2004, 333, 143-149.

(3) Esteller, M. CpG Island Hypermethylation and Tumor Suppressor Genes: A Booming Present, a Brighter Future. Oncogene 2002, 21, 5427-5440.

(4) Jones, P. A. Functions of DNA Methylation: Islands, Start Sites, Gene Bodies and Beyond. Nat. Rev. Genet. 2012, 13, 484-492.

(5) Schmid, Carl W and Prescott, L. D. Organization of the Human Genome Transcription. Cell 1975, 6, 345-358.

(6) Batzer, M. a; Deininger, P. L. Alu Repeats and Human Genomic Diversity. Nat. Rev. Genet. 2002, 3, 370-379.

(7) Mather, K. A.; Jorm, A. F.; Parslow, R. A.; Christensen, H. Is Telomere Length a Biomarker of Aging? A Review. J. Gerontol. A. Biol. Sci. Med. Sci. 2011, 66, 202-213.

(8) M. Duyao, C. Ambrose, R. Myers, A. Novelletto, F. Persichetti, M. Frontali, S. Folstein, C. Ross, M. Franz, M. Abbott, J. Gray, P. Conneally, A. Young, J. Penney, Z. Hollingsworth, I. Shoulson, A. Lazzarini, A. Falek, W. Koroshetz, D. Sax, E. Bird, J. Von, \& M. M. Trinucleotide Repeatlength Instability and Age of Onset in Huntington's Disease. Nat. Genet. 1993, 4, 387-392.

(9) Edwards, A.; Hammond, H. A.; Jin, L.; Caskey, C. T.; Chakraborty, R. Genetic Variation at Five Trimeric and Tetrameric Tandem Repeat Loci in Four Human Population Groups. Genomics 1992, 12, 241-253.

(10) Maarel, M. Van Der; Deidda, G.; Lemmers, R. J. L. F.; Overveld, P. G. M. Van; Wielen, M. Van Der; Hewitt, J. E.; Sandkuijl, L.; Bakker, B.; Ommen, G. B. Van; Padberg, G. W.; et al. De Novo Facioscapulohumeral Muscular Dystrophy $\square$ : Frequent Somatic Mosaicism, Sex-Dependent Phenotype , and the Role of Mitotic Transchromosomal Repeat Interaction between Chromosomes 4 and 10. 1996, 4, 26-35.

(11) Chamberlain, N. L.; Driver, E. D.; Miesfeld, R. L. The Length and Location of CAG Trinucleotide Repeats in the Androgen Receptor N-Terminal Domain Affect Transactivation Function. Nucleic Acids Res. 1994, 22, 3181-3186. 
(12) Balog, J.; Miller, D.; Sanchez-Curtailles, E.; Carbo-Marques, J.; Block, G.; Potman, M.; de Knijff, P.; Lemmers, R. J. L. F.; Tapscott, S. J.; van der Maarel, S. M. Epigenetic Regulation of the X-Chromosomal Macrosatellite Repeat Encoding for the Cancer/testis Gene CT47. Eur. J. Hum. Genet. 2012, $20,185-191$.

(13) Pook, M. A. DNA Methylation and Trinucleotide Repeat Expansion Diseases. In DNA Methylation - From Genomics to Technology; InTech, 2012; pp. 193208.

(14) Hansen, K. D.; Timp, W.; Bravo, H. C.; Sabunciyan, S.; Langmead, B.; McDonald, O. G.; Wen, B.; Wu, H.; Liu, Y.; Diep, D.; et al. Increased Methylation Variation in Epigenetic Domains across Cancer Types. Nat. Genet. 2011, 43, 768-775.

(15) Huichalaf, C.; Micheloni, S.; Ferri, G.; Caccia, R.; Gabellini, D. DNA Methylation Analysis of the Macrosatellite Repeat Associated with FSHD Muscular Dystrophy at Single Nucleotide Level. PLoS One 2014, 9, e115278.

(16) Gaillard, M.-C.; Roche, S.; Dion, C.; Tasmadjian, A.; Bouget, G.; SalortCampana, E.; Vovan, C.; Chaix, C.; Broucqsault, N.; Morere, J.; et al. Differential DNA Methylation of the D4Z4 Repeat in Patients with FSHD and Asymptomatic Carriers. Neurology 2014, 83, 733-742.

(17) Sacconi, S.; Lemmers, R. J. L. F.; Balog, J.; van der Vliet, P. J.; Lahaut, P.; van Nieuwenhuizen, M. P.; Straasheijm, K. R.; Debipersad, R. D.; Vos-Versteeg, M.; Salviati, L.; et al. The FSHD2 Gene SMCHD1 Is a Modifier of Disease Severity in Families Affected by FSHD1. Am. J. Hum. Genet. 2013, 93, 744751.

(18) INSERM and French Ministry of Health. Prevalence of Rare Diseases: Bibliographic Data http://www.orpha.net/orphacom/cahiers/docs/GB/Prevalence_of_rare_diseases _by_alphabetical_list.pdf.

(19) Korshunova, Y.; Maloney, R. K.; Lakey, N.; Citek, R. W.; Bacher, B.; Budiman, A.; Ordway, J. M.; McCombie, W. R.; Leon, J.; Jeddeloh, J. A.; et al. Massively Parallel Bisulphite Pyrosequencing Reveals the Molecular Complexity of Breast Cancer-Associated Cytosine-Methylation Patterns Obtained from Tissue and Serum DNA. Genome Res. 2008, 18, 19-29.

(20) Gu, H.; Smith, Z. D.; Bock, C.; Boyle, P.; Gnirke, A.; Meissner, A. Preparation of Reduced Representation Bisulfite Sequencing Libraries for Genome-Scale DNA Methylation Profiling. Nat. Protoc. 2011, 6, 468-481.

(21) Guo, H.; Zhu, P.; Wu, X.; Li, X.; Wen, L.; Tang, F. Single-Cell Methylome Landscapes of Mouse Embryonic Stem Cells and Early Embryos Analyzed Using Reduced Representation Bisulfite Sequencing. 2013, 2126-2135.

(22) Nagano, T.; Lubling, Y.; Stevens, T. J.; Schoenfelder, S.; Yaffe, E.; Dean, W.; Laue, E. D.; Tanay, A.; Fraser, P. Single-Cell Hi-C Reveals Cell-to-Cell Variability in Chromosome Structure. Nature 2013, 502, 59-64. 
(23) Treangen, T. J.; Salzberg, S. L. Repetitive DNA and next-Generation Sequencing: Computational Challenges and Solutions. Nat. Rev. Genet. 2012, $13,36-46$.

(24) Vranken, C.; Deen, J.; Dirix, L.; Stakenborg, T.; Dehaen, W.; Leen, V.; Hofkens, J.; Neely, R. K. Super-Resolution Optical DNA Mapping via DNA Methyltransferase-Directed Click Chemistry. Nucleic Acids Res. 2014, 42, e50.

(25) Nilsson, A. N.; Emilsson, G.; Nyberg, L. K.; Noble, C.; Svensson Stadler, L.; Fritzsche, J.; Moore, E. R. B.; Tegenfeldt, J. O.; Ambjörnsson, T.; Westerlund, F. Competitive Binding-Based Optical DNA Mapping for Fast Identification of Bacteria - Multi-Ligand Transfer Matrix Theory and Experimental Applications on Escherichia Coli. Nucleic Acids Res. 2014, 42, E118.

(26) Meng, X.; Benson, K.; Chada, K.; Huff, E. J.; Schwartz, D. C. Optical Mapping of Lambda Bacteriophage Clones Using Restriction Endonucleases. Nat. Genet. 1995, 9, 432-438.

(27) Levy-Sakin, M.; Ebenstein, Y. Beyond Sequencing: Optical Mapping of DNA in the Age of Nanotechnology and Nanoscopy. Curr. Opin. Biotechnol. 2013, $1-9$.

(28) Levy-Sakin, M.; Grunwald, A.; Kim, S.; Gassman, N. R.; Gottfried, A.; Antelman, J.; Kim, Y.; Ho, S. O.; Samuel, R.; Michalet, X.; et al. Toward Single-Molecule Optical Mapping of the Epigenome. ACS Nano 2014, 8, 1426.

(29) Cao, H.; Hastie, A. R.; Cao, D.; Lam, E. T.; Sun, Y.; Huang, H.; Liu, X.; Lin, L.; Andrews, W.; Chan, S.; et al. Rapid Detection of Structural Variation in a Human Genome Using Nanochannel-Based Genome Mapping Technology. Gigascience 2014, 3, 1-11.

(30) Mostovoy, Y.; Levy-Sakin, M.; Lam, J.; Lam, E. T.; Hastie, A. R.; Marks, P.; Lee, J.; Chu, C.; Lin, C.; Džakula, Ž.; et al. A Hybrid Approach for de Novo Human Genome Sequence Assembly and Phasing. Nat. Methods 2016, 12-17.

(31) Nifker, G.; Levy-Sakin, M.; Berkov-Zrihen, Y.; Shahal, T.; Gabrieli, T.; Fridman, M.; Ebenstein, Y. One-Pot Chemoenzymatic Cascade for Labeling of the Epigenetic Marker 5-Hydroxymethylcytosine. Chembiochem 2015, 16, 1857-1860.

(32) Zirkin, S.; Fishman, S.; Sharim, H.; Michaeli, Y.; Don, J.; Ebenstein, Y. Lighting up Individual DNA Damage Sites by in Vitro Repair Synthesis. J. Am. Chem. Soc. 2014, 136, 7771-7776.

(33) Michaeli, Y.; Shahal, T.; Torchinsky, D.; Grunwald, A.; Hoch, R.; Ebenstein, Y. Optical Detection of Epigenetic Marks: Sensitive Quantification and Direct Imaging of Individual Hydroxymethylcytosine Bases. Chem. Commun. (Camb). 2013, 49, 8599-8601.

(34) Gilboa, T.; Torfstein, C.; Juhasz, M.; Grunwald, A.; Ebenstein, Y.; Weinhold, E.; Meller, A. Single-Molecule DNA Methylation Quantification Using Electro-Optical Sensing in Solid-State Nanopores. ACS Nano 2016, 10, 8861- 
8870.

(35) GeneCards - Human Genes | Gene Database | Gene Search http://www.genecards.org/.

(36) Kinkley, S.; Staege, H.; Mohrmann, G.; Rohaly, G.; Schaub, T.; Kremmer, E.; Winterpacht, A.; Will, H. SPOC1: A Novel PHD-Containing Protein Modulating Chromatin Structure and Mitotic Chromosome Condensation. J. Cell Sci. 2009, 122.

(37) Klimasauskas, S.; Weinhold, E. A New Tool for Biotechnology: AdoMetDependent Methyltransferases. Trends Biotechnol. 2007, 25, 99-104.

(38) Hanz, G. M.; Jung, B.; Giesbertz, A.; Juhasz, M.; Weinhold, E. SequenceSpecific Labeling of Nucleic Acids and Proteins with Methyltransferases and Cofactor Analogues. J. Vis. Exp. 2014, 3-12.

(39) Dalhoff, C.; Weinhold, E.; Klimas, S. Direct Transfer of Extended Groups from Synthetic Cofactors by DNA Methyltransferases. 2006, 2, 2005-2006.

(40) McClelland, M.; Nelson, M. Effect of Site-Specific Méthylation on Dna Modification Methyltransferases and Restriction Endonucleases. Nucleic Acids Res. 1992, 20, 2145-2157.

(41) Hansen, K. D.; Sabunciyan, S.; Langmead, B.; Nagy, N.; Curley, R.; Klein, G.; Klein, E.; Salamon, D.; Feinberg, A. P. Large-Scale Hypomethylated Blocks Associated with Epstein-Barr Virus - Induced B-Cell Immortalization. 2014, 177-184.

(42) Das, S. K.; Austin, M. D.; Akana, M. C.; Deshpande, P.; Cao, H.; Xiao, M. Single Molecule Linear Analysis of DNA in Nano-Channel Labeled with Sequence Specific Fluorescent Probes. Nucleic Acids Res. 2010, 38, e177.

(43) Kriukienè, E.; Labrie, V.; Khare, T.; Urbanavičiūte, G.; Lapinaitè, A.; Koncevičius, K.; Li, D.; Wang, T.; Pai, S.; Ptak, C.; et al. DNA Unmethylome Profiling by Covalent Capture of CpG Sites. Nat. Commun. 2013, 4, 916-919.

(44) Reinius, L. E.; Acevedo, N.; Joerink, M.; Pershagen, G.; Dahlén, S. E.; Greco, D.; Söderhäll, C.; Scheynius, A.; Kere, J. Differential DNA Methylation in Purified Human Blood Cells: Implications for Cell Lineage and Studies on Disease Susceptibility. PLoS One 2012, 7.

(45) Cabianca, D. S.; Gabellini, D. The Cell Biology of Disease: FSHD: Copy Number Variations on the Theme of Muscular Dystrophy. J. Cell Biol. 2010, $191,1049-1060$.

(46) Yoon, S.; Xuan, Z.; Makarov, V.; Ye, K.; Sebat, J. Sensitive and Accurate Detection of Copy Number Variants Using Read Depth of Coverage. Genome Res. 2009, 19, 1586-1592.

(47) Thomas Anantharaman, B. M. False Positives in Genomic Map Assembly and Sequence Validation; Gascuel, O.; Moret, B. M. E., Eds.; Lecture Notes in Computer Science; Springer Berlin Heidelberg: Berlin, Heidelberg, 2001; Vol. 2149. 
(48) Pendleton, M.; Sebra, R.; Pang, A. W. C.; Ummat, A.; Franzen, O.; Rausch, T.; Stütz, A. M.; Stedman, W.; Anantharaman, T.; Hastie, A.; et al. Assembly and Diploid Architecture of an Individual Human Genome via Single-Molecule Technologies. Nat. Methods 2015, 12, 780-786.

(49) Jeffet, J.; Kobo, A.; Su, T.; Grunwald, A.; Green, O.; Nilsson, A. N.; Eisenberg, E.; Ambjörnsson, T.; Westerlund, F.; Weinhold, E.; et al. SuperResolution Genome Mapping in Silicon Nanochannels. ACS Nano 2016, 10, 9823-9830.

(50) Lemmers, R. J. L. F.; Wohlgemuth, M.; van der Gaag, K. J.; van der Vliet, P. J.; van Teijlingen, C. M. M.; de Knijff, P.; Padberg, G. W.; Frants, R. R.; van der Maarel, S. M. Specific Sequence Variations within the 4q35 Region Are Associated with Facioscapulohumeral Muscular Dystrophy. Am. J. Hum.

Genet. 2007, 81, 884-894.

(51) Lemmers, R. J. L. F.; Tawil, R.; Petek, L. M.; Balog, J.; Block, G. J.; Santen, G. W. E.; Amell, A. M.; van der Vliet, P. J.; Almomani, R.; Straasheijm, K. R.; et al. Digenic Inheritance of an SMCHD1 Mutation and an FSHD-Permissive D4Z4 Allele Causes Facioscapulohumeral Muscular Dystrophy Type 2. Nat. Genet. 2012, 44, 1370-1374.

(52) Choi, S. H.; Worswick, S.; Byun, H.-M.; Shear, T.; Soussa, J. C.; Wolff, E. M.; Douer, D.; Garcia-Manero, G.; Liang, G.; Yang, A. S. Changes in DNA Methylation of Tandem DNA Repeats Are Different from Interspersed Repeats in Cancer. Int. J. Cancer 2009, 125, 723-729.

(53) Grunwald, A.; Dahan, M.; Giesbertz, A.; Nilsson, A.; Nyberg, L. K.; Weinhold, E.; Ambjörnsson, T.; Westerlund, F.; Ebenstein, Y. Bacteriophage Strain Typing by Rapid Single Molecule Analysis. Nucleic Acids Res. 2015, 18 .

(54) Arielly, R.; Ebenstein, Y. Irys Extract. Bioinformatics 2017, 136, 7771-7776.

(55) Kent, W. J.; Sugnet, C. W.; Furey, T. S.; Roskin, K. M. The Human Genome Browser at UCSC W. J. Med. Chem. 1976, 19, 1228-1231. 